\title{
Comparative study between Ephedra sinica Stapf and Fructus Schisandrae Chinensis on ET-1 and 6-keto-prostaglandin F1 $\alpha$ in rats with idiopathic pulmonary fibrosis
}

\author{
H.Q. Zhai ${ }^{1,2}$, J.R. Hhang ${ }^{3}$, M.C. Gao ${ }^{1}$, Y. Liu ${ }^{1}$, S.F. Zhang ${ }^{1}$, X.H. Wang ${ }^{1}$, \\ F.Y. Meng ${ }^{2}$ and Y.P. Wang ${ }^{4}$ \\ ${ }^{1}$ Institute of Natural Medicine and Chinese Medicine Resources, \\ Beijing Normal University, Beijing, China \\ ${ }^{2}$ School of Chinese Pharmacy, \\ Beijing University of Chinese Medicine, Beijing, China \\ ${ }^{3}$ Medicine School, Yangtze University, Jingzhou, China \\ ${ }^{4}$ China Academy of Traditional Chinese Medicine, Beijing, China \\ Corresponding author: H.Q. Zhai \\ E-mail: huaqiangzhai@126.com
}

Genet. Mol. Res. 13 (2): 3761-3771 (2014)

Received August 10, 2013

Accepted December 15, 2013

Published May 13, 2014

DOI http://dx.doi.org/10.4238/2014.May.13.3

\begin{abstract}
The aim of this study was to evaluate differences in the effects of Ephedra sinica Stapf and Fructus Schisandrae Chinensis on angiogenesis in the treatment of bleomycin-induced rat idiopathic pulmonary fibrosis. The rat models were created using bleomycin. The animals were divided into six groups: model, control, Ephedra alone, Schisandrae alone, combination of Ephedra and Schisandrae, and hydrocortisone alone. The treatments were administered for 28 days. After 7 and 28 days, the rats were sacrificed for pathological morphology examination, microvascular density determination,
\end{abstract}


and angiogenesis-related cytokine examination. The Ephedra and hydrocortisone groups demonstrated significantly reduced alveolitis and pulmonary fibrosis grades compared with the model group $(\mathrm{P}<$ $0.05)$. The number of blood vessels in the Ephedra group was higher than that in the Schisandrae and combination therapy groups. At 7 days, the expression level of endothelin (ET)-1 in the model group was significantly higher than that in the normal group $(\mathrm{P}<0.01)$. The level of 6-keto-prostaglandin F1 $\alpha$ (6-keto-PGF1 $\alpha)$ in the treatment group increased, and there were significant differences between the Ephedra group and the combination therapy and normal groups $(\mathrm{P}<0.05)$. Ephedra inhibited the increase in the lung coefficient. The combination therapy prevented pulmonary artery injury and angiogenesis of the arteries by reducing the level of ET-1 and promoting the level of 6-keto-PGF1 $\alpha$ in the blood. Ephedra and Schisandrae prevented alveolitis and the development of pulmonary fibrosis.

Key words: Ephedra sinica Stapf; Fructus Schisandrae Chinensis; Endothelin-1; Idiopathic pulmonary fibrosis

\section{INTRODUCTION}

Epidemiological surveys have demonstrated that idiopathic pulmonary fibrosis (IPF) is the most difficult disease to treat globally. The morbidity has increased in recent years and IPF has become a common clinical disease (Tzortzaki et al., 2007; Meltzer and Noble, 2008). At the onset of the disease, the respiratory system is affected; however, as the disease progresses, more systems and major organs become affected. The prognosis is poor and the mortality of the disease increases with age. IPF mainly occurs in male patients, affecting their working capacity and quality of life. The pathogenesis is currently unknown, and therefore therapeutic methods for IPF are not specific. The current treatment methods are anti-inflammatory drug therapy, immunosuppressive and immunomodulatory agents, anti-fibrosis drugs, antioxidants, anti-leukotriene drugs, and lung transplantation; however, these treatments are associated with controversy, and large sample epidemiological surveys have shown that their efficacies are not exact. In recent years, the study of the pathogenesis of IPF has no longer been confined to the pulmonary alveolitis mechanism (Unemori et al., 1996; Hirose et al., 2000; Strieter et al., 2002), as more studies increasingly concentrate on vascular injury and the mechanism of angiogenesis. A large number of clinical studies have demonstrated that traditional Chinese medicine effectively relieves various symptoms of pulmonary fibrosis and delays the progression of the disease (Yao and Jiang, 2005; Song and Liu, 2007; Zhao and Yin, 2008; Li and Li, 2009). A series of experiments have shown that traditional Chinese medicine improves abnormal indicators and the performance of the pathological mechanism affected by pulmonary fibrosis. It is important to confirm that traditional Chinese medicine has a major impact on vascular injury and angiogenesis. Ephedra and Schisandrae have been used to treat pulmonary fibrosis in a clinical setting. Ephedra is used as an ascending-floating drug and Schisandrae is used as a descending- 
sinking drug; however, their mechanisms of action have not yet been elucidated. Our study not only clarifies this mechanism, but also provides innovative ideas and methods for the ascending-descending-floating-sinking theory.

\section{MATERIAL AND METHODS}

\section{Modeling method}

Ninety male specific pathogen-free Wistar rats aged 6 weeks and weighing $200 \pm 15$ g were provided by the Beijing Vital River Laboratory Animal Technology Co., Ltd. [permission No. SCXK 2006-0009]. The rats were housed at a controlled temperature $\left(25^{\circ} \pm 1^{\circ} \mathrm{C}\right)$ with a relative humidity of $50-60 \%$. Rats had free access to food and tap water. All animals used in the study received humane care in compliance with institutional animal care guidelines. The study was carried out in strict accordance with the recommendations in the Guide for the Care and Use of Laboratory Animals of the National Institutes of Health. The animal use protocol was reviewed and approved by the Institutional Animal Care and Use Committee (IACUC) of the Sun Beijing University of Chinese Medicine.

The drugs tested were as follows: Ephedra decoction (prepared by boiling Ephedra sinica Stapf 8 and 4 times in water for $30 \mathrm{~min}$ and $20 \mathrm{~min}$, respectively, concentrated at $1 \mathrm{~g}$ crude drug/mL, and stored at $4^{\circ} \mathrm{C}$ ), Schisandrae decoction (prepared from Fructus Schisandrae Chinensis by a method identical to that of the Ephedra decoction), and injection of $20 \mathrm{mg} / \mathrm{kg}$ control drug, hydrocortisone (Shanxi Jinxin Shuanghe Pharmaceutical Co., Ltd.; Yuncheng, China; approval No. H14020980; batch No. 20080917).

To create the rat model, hair was removed from the neck and an intraperitoneal injection of $30 \mathrm{mg} / \mathrm{kg} 1 \%$ pentobarbital sodium was administered to anesthetize the rats. They were then fixed in the supine position on a rat board covered with medical towels and the skin was disinfected with $75 \%$ alcohol. The skin was cut $2-3 \mathrm{~cm}$ on the neck midline and the trachea was exposed using vessel forceps. A No. 4 needle was passed through the tracheal cartilage ring to the end of the trachea, and $7 \mathrm{mg} / \mathrm{kg}$ bleomycin hydrochloride was quickly injected into the trachea. The rat board was immediately placed upright and rotated 100 turns, and then placed vertically for $3 \mathrm{~min}$ to ensure a uniform drug distribution in the lungs. A suture needle was then used to suture the muscle and skin. The area was resterilized and an intraperitoneal injection of $80,000 \mathrm{U} /$ day sodium penicillin was administered to prevent infection. The normal group underwent the same modeling method except that $0.9 \%$ physiological saline was injected into the trachea instead of bleomycin hydrochloride.

\section{Drug administration}

The animals were randomly divided into six groups with 10 animals in the normal group and 16 animals in each of the other groups. After modeling, the normal group was given 6 $\mathrm{mL} \cdot \mathrm{kg}^{-1} \cdot \mathrm{day}^{-1}$ normal saline from day 1-28; the model group was given $6 \mathrm{~mL} \cdot \mathrm{kg}^{-1} \cdot \mathrm{day}^{-1}$ normal saline from day 1-28; the Ephedra group was treated with $5 \mathrm{~mL} \cdot \mathrm{kg}^{-1} \cdot$ day $^{-1}$ Ephedra decoction concentrate from day 1-28; the Schisandrae group was treated with $5 \mathrm{~mL} \cdot \mathrm{kg}^{-1} \cdot \mathrm{day}^{-1}$ Schisandrae decoction concentrate from day 1-28; the combination therapy group was treated with 6 $\mathrm{mL} \cdot \mathrm{kg}^{-1} \cdot$ day $^{-1}$ Ephedra decoction concentrate from day $1-7$ and $5 \mathrm{~mL} \cdot \mathrm{kg}^{-1} \cdot$ day $^{-1}$ Schisandrae 
decoction from day 7-28; and the hydrocortisone group received $20 \mathrm{mg} \cdot \mathrm{kg}^{-1} \cdot \mathrm{day}^{-1}$ hydrocortisone therapy from day 1-28 by subcutaneous injection.

\section{Lung coefficient measurement}

The rats were sacrificed 7 and 28 days after drug administration by abdominal aortic bleeding. The skin was cut along the midline of the abdominal wall, exposing the abdominal organs. The intestines were moved to the right side, and the fat at the front of the spine was cleaned and separated using cotton yarn to expose the abdominal aorta. Using a No. 4 sterile needle, the abdominal aorta was pierced to collect $5 \mathrm{~mL}$ blood. Immediately afterward, the thoracic ribs were cut open and the rats died from loss of blood. The lungs were then removed using surgical scissors and forceps and were placed in sterile saline at $24^{\circ} \mathrm{C}$. Lungs were rinsed repeatedly to wash away the blood until the tissue appeared white. The lungs' weights were recorded using an electronic balance. The clean lungs were placed in $10 \%$ neutral formaldehyde solution and fixed for $24 \mathrm{~h}$ ready for inspection. The following equation was used to calculate the lung coefficient: lung coefficient = lung weight / body weight $\times 100$.

\section{Pathological morphology examination}

The testing methods and procedures were as follows: i) the tissues were taken, embedded in paraffin, and sliced to $5 \mu \mathrm{m}$ thickness; ii) the sections were dipped in $50^{\circ} \mathrm{C}$ xylene I followed by xylene II, each for $25 \mathrm{~min}$, to remove the wax, and the xylene was then removed using $100 \%$ alcohol followed by $95,90,80$, and $75 \%$ alcohol, each for $1 \mathrm{~min}$; iii) washed with water for $2 \mathrm{~min}$; iv) stained with sappan wood liquid for $2 \mathrm{~min}$ and then washed with tap water for $1 \mathrm{~min}$; v) differentiated using $0.5 \%$ hydrochloric acid ethanol liquid for $2 \mathrm{~s}$; vi) washed fully with tap water once, and then washed twice with distilled water; vii) treated with $1 \%$ dilute ammonia for a few seconds and then washed with water for $1 \mathrm{~min}$; viii) eosin stained for $2 \mathrm{~min}$; ix) washed fully with tap water; $\mathrm{x}$ ) dehydrated (85\% alcohol for $20 \mathrm{~s}, 90 \%$ alcohol for $30 \mathrm{~s}, 95 \%$ alcohol for $1 \mathrm{~min}$, and $100 \%$ alcohol for $2 \mathrm{~min}$ ); xi) cleared in two changes of xylene for 2 min each; xii) cemented with neutral gum, and dried.

Observations were made using an ordinary light microscope. Hematoxylin and eosin (H\&E) staining was used to evaluate pathological changes in lung tissues. The alveolitis classification was as follows: no alveolitis (-), mild alveolitis (+; affected area $<20 \%)$, moderate alveolitis (++; affected area $\sim 20-50 \%$ ), and severe alveolitis (+++; affected area $>50 \%$ ). The classification of pulmonary fibrosis was as follows: no pulmonary fibrosis (-), light degree of pulmonary fibrosis ( + ; lesion range $<20 \%$ in the whole lung), moderate pulmonary fibrosis (++; lesion range $\sim 20-50 \%$ in the whole lung), and severe pulmonary fibrosis (+++; lesion range $>50 \%$ in the whole lung, accompanied by alveolar fusion and lung parenchyma structural disorder). The nuclear density (ND) and external diameter (ED) were also calculated: ND $=$ number of nuclei / medial area.

\section{Microvascular density (MVD) examination}

The specimen histotomy and testing method were the same as described above. MVD was measured as follows: immunohistochemical methods were used on the embedded lung 
biopsy specimens to visualize factor VIII-related antigen. Magnification (5 x 10) allowed determination of the area with the highest concentration of capillaries. In this area, the chromatic blood vessel number and the deep brown and tan endothelial cell number were counted in five consecutive microscopic fields of vision (magnification, $40 \times 10$ ). A number of microscopic measurements of microvasculature were used to calculate the blood vessel density $\left(\right.$ per $\mathrm{mm}^{2}$ ) in each field of vision. The MVD was determined as the average of these measurements (Weidner, 1995).

\section{Angiogenesis-related cytokine examination}

Blood samples were sent to Beijing Biological Engineering Co. (Beijing, China) for determination of cytokine levels.

The equipment and instruments used were as follows: an FT-646A single-head computer radioimmunoassay instrument (Beijing Nuclear Instrument Factory; Beijing, China), a GC-1200r RIA counter (University of Science and Technology of China; Anhui, China), and a $2500 \mathrm{rpm}$ LXJ-II-type centrifugal sedimentation machine (Shanghai Medical Instrument Factory; Shanghai, China).

\section{Statistical analysis}

Data are reported as means \pm standard deviation. Measurement data were analyzed by analysis of variance and the Student $t$-test. Counted data were analyzed by the chi-squared test, and for comparison between groups, multifactorial analysis of variance was used. $\mathrm{P}<0.05$ was considered to indicate a statistically significant difference. Statistical analyses were performed using the SPSS 11.0.0 software (SPSS Inc.; Chicago, IL, USA).

\section{Results}

\section{Lung coefficient in IPF rats}

The lung coefficient of the model group increased significantly compared with that of the normal group $(\mathrm{P}<0.05)$. The lung coefficients of the Ephedra and model groups were significantly different $(\mathrm{P}<0.05)$. At 28 days after surgery, no significant difference was observed between the lung coefficients of the Ephedra and Schisandrae groups $(\mathrm{P}>0.05$; Table 1 and Figure 1).

Table 1. Lung coefficient in idiopathic pulmonary fibrosis rats.
\begin{tabular}{lccc}
\hline Group & $\mathrm{N}$ & 7 days $(\%)$ & 28 days (\%) \\
\hline Ephedra group & 16 & $4.613 \pm 0.336^{*}$ & $5.476 \pm 0.243$ \\
Schisandrae group & 16 & $4.746 \pm 0.941$ & $4.793 \pm 0.388$ \\
Combination therapy group & 16 & 0 & $4.823 \pm 0.352$ \\
Hydrocortisone group & 16 & $6.660 \pm 0.334$ & $4.467 \pm 0.107$ \\
Model group & 16 & $8.631 \pm 0.674$ & $5.591 \pm 0.111$ \\
Normal group & 10 & $4.381 \pm 0.429^{* *}$ & $4.055 \pm 0.106^{*}$ \\
\hline Data are reported as means \pm SD. ${ }^{*} \mathrm{P}<0.05 ; * * \mathrm{P}<0.01$ compared with model group.
\end{tabular}




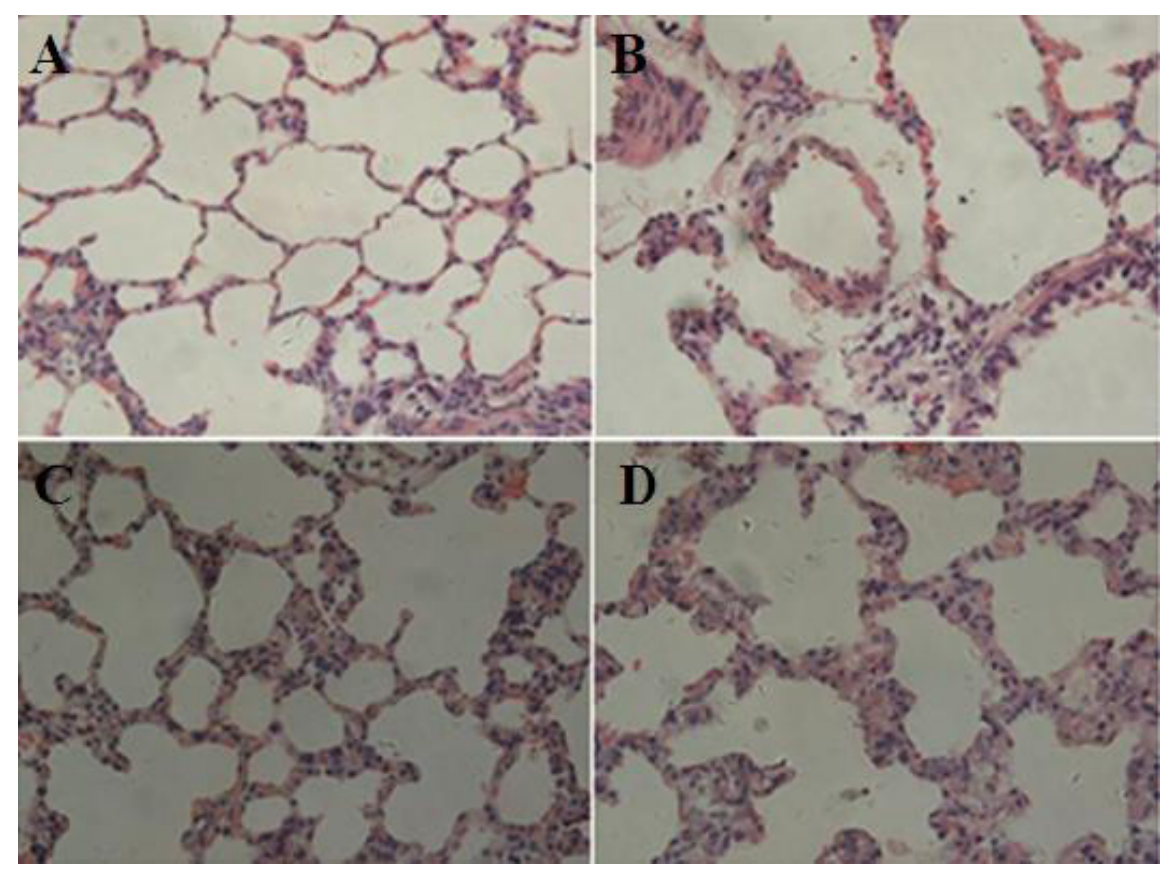

Figure 1. Hematoxylin-eosin staining of the pulmonary tissue 28 days after surgery (light microscopy, 400X). A. Normal control group; B. Model group; C. Ephedra sinica Stapf group; D. Fructus Schisandrae Chinensis group.

\section{Lung tissue in IPF rats by H\&E staining}

The normal group demonstrated a clear lung structure, alveolar structural integrity, no fusion or defects, no thickening of the pulmonary interstitium, no fiber bundle formation, no alveolar exudate, pulmonary vascular structural integrity, and no inflammation. The model group presented inflammatory cell infiltration in the visual field, and 7 days after surgery, there was scattered alveolar damage, fusion, bullae formation, an accumulation of alveolar exudate, and thickening of the pulmonary interstitial filling, but no obvious fibrous connective tissue bundles. Compared with 7 days after modeling, the degree of inflammatory cell infiltration was slightly reduced at 28 days, and alveolar wall damage and integration and formation of multiple bullae were observed. There were large numbers of fibrous connective tissue bundles in the pulmonary interstitium, with bronchial lesions being the most common. Other treatment groups presented a small amount of alveolar inflammatory cell infiltration, defective alveolar structures, and the integration and formation of bullae. Alveolar exudation was observed to varying degrees but was lighter than that in the model group. Pulmonary interstitial fibrous connective tissue formation was also noted to varying degrees, with bronchial lesions being the most common. Occasional capillary wall membrane defects were also observed. Significant differences were identified between the normal and model groups 7 and 28 days after surgery. The extent of alveolitis and bleomycin-induced IPF in the model group was more severe than in the normal group $(\mathrm{P}<0.01)$. After 7 days, the hydrocortisone and model groups demonstrated a significant difference $(\mathrm{P}<0.05)$, and after 28 days, significant differences were 
observed among the combination therapy, hydrocortisone, and model groups. The combination therapy and the hydrocortisone treatment significantly prevented alveolitis and IPF in the rats $(\mathrm{P}<0.05$; Table 2$)$.

\begin{tabular}{|c|c|c|c|c|c|c|c|c|c|c|}
\hline \multirow[t]{2}{*}{ Group } & \multicolumn{5}{|c|}{7 days alveolar inflammation } & \multicolumn{5}{|c|}{28 days fibrosis classification } \\
\hline & $\mathrm{N}$ & $(-)$ & $(+)$ & $(++)$ & $(+++)$ & $\mathrm{N}$ & $(-)$ & $(+)$ & $(++)$ & $(+++)$ \\
\hline Ephedra group & 8 & 0 & 2 & 3 & 3 & 8 & 0 & 2 & 3 & 3 \\
\hline Schisandrae group & 8 & 0 & 2 & 2 & 4 & 8 & 0 & 3 & 1 & 4 \\
\hline Combination therapy group & 8 & 0 & 0 & 0 & 0 & 8 & $0^{*}$ & 2 & 4 & 2 \\
\hline Hydrocortisone group & 8 & $0^{*}$ & 3 & 3 & 2 & 8 & $0^{*}$ & 5 & 2 & 1 \\
\hline Model group & 8 & 0 & 1 & 2 & 5 & 8 & 0 & 1 & 2 & 5 \\
\hline Normal group & 5 & $4 * *$ & 1 & 0 & 0 & 5 & $4 * *$ & 1 & 0 & 0 \\
\hline
\end{tabular}

**P $<0.01 ; * \mathrm{P}<0.05$ compared with model group.

\section{ND, ED, and MVD in IPF rats}

The normal, combination therapy, and hydrocortisone groups all demonstrated a significant difference from the model group in ND and ED $(\mathrm{P}<0.05)$. There were no significant differences in ND and ED between the Ephedra, Schisandrae, and model groups ( $\mathrm{P}>0.05$; Tables 3 and 4).

\begin{tabular}{llc} 
Table 3. Nuclear density (ND) in idiopathic pulmonary fibrosis rats. & ND $\left(/ \mu \mathrm{m}^{2}\right)$ \\
\hline Group & $\mathrm{N}$ & $0.01246 \pm 0.00493$ \\
\hline Ephedra group & 16 & $0.01158 \pm 0.00502$ \\
Schisandrae group & 16 & $0.01143 \pm 0.00362^{*}$ \\
Combination therapy group & 16 & $0.01107 \pm 0.00368^{*}$ \\
Hydrocortisone group & 16 & $0.01656 \pm 0.00103$ \\
Model group & 16 & $0.01092 \pm 0.00494^{*}$ \\
Normal group & 10 &
\end{tabular}

Data are reported as means $\pm \mathrm{SD} .{ }^{*} \mathrm{P}<0.05$ compared with model group.

\begin{tabular}{llc}
\multicolumn{2}{c}{ Table 4. External diameter (ED) in idiopathic pulmonary fibrosis rats. } \\
\hline Group & $\mathrm{N}$ & $\mathrm{ED}(\mu \mathrm{m})$ \\
\hline Ephedra group & 16 & $77.60 \pm 16.53$ \\
Schisandrae group & 16 & $71.86 \pm 20.52$ \\
Combination therapy group & 16 & $86.11 \pm 14.52 *$ \\
Hydrocortisone group & 16 & $90.86 \pm 21.11 *$ \\
Model group & 16 & $49.35 \pm 11.25$ \\
Normal group & 10 & $88.41 \pm 11.32 *$ \\
\hline
\end{tabular}

Data are reported as means $\pm \mathrm{SD} . * \mathrm{P}<0.05$ compared with model group.

The number of blood vessels in the model group was significantly higher than that in the normal group $(\mathrm{P}<0.05)$. The Ephedra treatment was significantly weaker than the combination therapy in restraining vessel proliferation in the lung. The number of blood vessels in the Ephedra group was higher than those in the Schisandrae and combination therapy groups $(\mathrm{P}<0.05$; Table 5). 
Table 5. Number of blood vessels of rats in different groups.

\begin{tabular}{llc}
\hline Group & $\mathrm{N}$ & Number of blood vessels $\left(\mathrm{mm}^{2}\right)$ \\
\hline Ephedra group & 16 & $11.53 \pm 1.51^{*}$ \\
Schisandrae group & 16 & $9.31 \pm 1.36^{*}$ \\
Combination therapy group & 16 & $9.42 \pm 0.43^{*}$ \\
Hydrocortisone group & 16 & $7.75 \pm 1.76^{*}$ \\
Model group & 16 & $17.10 \pm 1.30$ \\
Normal group & 10 & $6.44 \pm 0.53^{*}$ \\
\hline
\end{tabular}

Data are reported as means $\pm \mathrm{SD} . * \mathrm{P}<0.05$ compared with model group.

\section{6-Keto-prostaglandin F1 $\alpha$ (6-keto-PGF1 $\alpha$ ) and endothelin (ET)-1 in IPF rats}

The ET-1 level in the normal group was significantly lower than that in the model group 28 days after surgery $(\mathrm{P}<0.05)$. The ET-1 levels of the hydrocortisone and Ephedra groups were also significantly different from the model group $(\mathrm{P}<0.05$; Table 6$)$.

Table 6. Endothelin-1 in idiopathic pulmonary fibrosis rats.
\begin{tabular}{lccc}
\hline Group & $\mathrm{N}$ & 7 days $(\mathrm{pg} / \mathrm{mL})$ & 28 days $(\mathrm{pg} / \mathrm{mL})$ \\
\hline Ephedra group & 16 & $83.91 \pm 13.93$ & $85.36 \pm 15.40^{*}$ \\
Schisandrae group & 16 & $85.36 \pm 18.93$ & $89.20 \pm 12.07$ \\
Combination therapy group & 16 & 0 & $82.97 \pm 14.26$ \\
Hydrocortisone group & 16 & $76.68 \pm 5.13$ & $79.29 \pm 13.92^{*}$ \\
Model group & 16 & $110.09 \pm 23.32$ & $106.76 \pm 33.49$ \\
Normal group & 10 & $73.88 \pm 17.26^{*}$ & $75.77 \pm 11.43^{* *}$ \\
\hline
\end{tabular}

Data are reported as means $\pm \mathrm{SD} .{ }^{*} \mathrm{P}<0.05 ; * * \mathrm{P}<0.01$ compared with model group.

Prior to treatment, no significant differences in 6-keto-PGF1 $\alpha$ levels were observed among any of the groups $(\mathrm{P}>0.05)$. Following treatment, however, a significant difference was observed between the combination therapy group and the model group $(\mathrm{P}<0.05$; Table 7$)$.

Table 7. 6-Keto-prostaglandin F1 $\alpha$ in idiopathic pulmonary fibrosis rats.

\begin{tabular}{llcc}
\hline Group & $\mathrm{N}$ & 7 days $(\mathrm{pg} / \mathrm{mL})$ & 28 days $(\mathrm{pg} / \mathrm{mL})$ \\
\hline Ephedra group & 16 & $118.84 \pm 20.14$ & $128.35 \pm 15.29$ \\
Schisandrae group & 16 & $83.38 \pm 14.45$ & $110.07 \pm 13.90$ \\
Combination therapy group & 16 & 0 & $143.57 \pm 19.74 *$ \\
Hydrocortisone group & 16 & $119.67 \pm 24.10$ & $128.37 \pm 29.47$ \\
Model group & 16 & $93.13 \pm 18.69$ & $75.73 \pm 18.69$ \\
Normal group & 10 & $119.09 \pm 25.69$ & $114.70 \pm 21.56$ \\
\hline
\end{tabular}

Data are reported as means $\pm \mathrm{SD} . * \mathrm{P}<0.05$ compared with model group.

\section{DISCUSSION}

The clinical application of traditional Chinese medical herbs is continuously being improved (Snider et al., 1978; Szapiel et al., 1979; Lohmann-Matthes et al., 1994; Rubins, 2003). The use of traditional Chinese medical herbs for the treatment of lung diseases has been shown of great value; however, it requires an understanding of the physiological characteristics of pulmonary medicine, abnormalities in pulmonary diseases, and the effects of a drug on 
pathogenesis in the lungs. Traditional Chinese medicines have shown positive effects in the treatment of common diseases, including pulmonary fibrosis. Research on IPF and its treatment must be taken seriously with consideration of several major issues. Impaired depurative descending of lung coefficient is the key pathogenesis in pulmonary fibrosis (Zhai et al., 2010) and it features throughout the course of the disease. In Chinese medicine, a full understanding of the features of pathogenesis and rational drug adjustment are the keys to achieving good efficacy in the treatment of lung fibrosis.

Pulmonary fibrosis is characterized by early lung injury followed by fibrosis. In our model, these two processes appeared at different time points: at 7 days, the main manifestation was alveolitis, and then at 28 days, significant fibrosis appeared (Zheng et al., 2000; Lu et al., 2001; Ye et al., 2008). The current study identified that the model group had significant lung inflammation and fibrosis at 7 days. Compared with the model group, fibrosis in the combination therapy group significantly improved, while the Ephedra and Schisandrae groups showed no significant improvement. ND and ED are the best parameters for measuring pathological changes in the lung arteriole in the process of pulmonary fibrosis (Keane et al., 2002). ND reflects the activity of vascular smooth muscle cells (VSMCs). We observed that the NDs of the normal and model groups were significantly different $(\mathrm{P}<0.05)$, which indicates that the process of pulmonary fibrosis causes significant pulmonary arterial hyperplasia. Additionally, the ND in the hydrocortisone group was significantly different from that in the model group $(\mathrm{P}<0.05)$, indicating that hydrocortisone inhibits capillary proliferation. The observation that the ND in the combination therapy group was significantly different from that in the model group $(\mathrm{P}<0.05)$ also indicates that combination therapy has an inhibitory effect on VSMC. This inhibition did not occur in the rats treated with Ephedra or Schisandrae alone.

The ED reflects the tension and proliferative state of the small blood vessels in the lungs (Syeda et al., 2006). The EDs in the normal and model groups were significantly different $(\mathrm{P}<0.05)$, which suggests that pulmonary fibrosis is accompanied by pulmonary capillary proliferation, resulting in stenosis of the lumen. This stenosis causes insufficient blood supply to the lung tissue, which may be the mechanism of promoting the membrane cell division irritability acceleration. The EDs in the combination therapy and model groups were significantly different $(\mathrm{P}<0.05)$, which indicates the remission effect of the combination treatment on pulmonary fibrosis. Vessel tension and proliferation did not occur in the Ephedra and Schisandrae groups. This study also demonstrated that the MVD in the model group was significantly different from that in the normal group $(\mathrm{P}<0.05)$, which indicates that vessel neogenesis occurs in pulmonary fibrosis. The Ephedra group demonstrated significant differences from the normal and hydrocortisone groups $(\mathrm{P}<0.05)$. This indicates that the effect of Ephedra is less potent than that of hydrocortisone.

The current study found that at 7 days, all treatment groups, with the exception of the Schisandrae group, had a higher concentration of plasma 6-keto-PGF1 $\alpha$ than the model group, which was similar to the concentration of the normal group. Produced in vascular endothelial cells, 6-keto-PGF1 $\alpha$ inhibits platelet aggregation and promotes vasodilation, and is therefore an important factor in vascular endothelial injury. Vascular endothelial injury may lead to a reduction of 6-keto-PGF1 $\alpha$ levels; however, no significant difference was observed between the groups. This indicates that vascular endothelial injury occurred in the IPF rats. In the model group, 6-keto-PGF1 $\alpha$ levels continued to decline 28 days after surgery. In the treatment groups, 6-keto-PGF1 $\alpha$ levels were higher than the normal level, which indicated 
that vascular endothelial injury in the treatment groups had improved. Additionally, at 7 days, the plasma ET-1 level in the normal group was significantly different from that in the model group. In the model group, the rat lung tissue experienced pulmonary fibrosis; therefore, ET-1 acted on receptors on the small pulmonary arteries, causing contraction of the small arteries, thus increasing the pathological state of tissue ischemia and hypoxia. The treatment groups demonstrated different plasma ET-1 levels compared with the model group; however, this difference was not significant after 7 days, indicating that the ET-1-induced pulmonary fibrosis was not effectively controlled. At 28 days, the normal group displayed a significant difference in plasma ET-1 levels when compared with the model group. This difference was due to the increase in the plasma ET-1 level in the model group throughout the whole process of pulmonary fibrosis. In the Ephedra and hydrocortisone groups, the plasma ET-1 levels increased slightly; however, they remained similar to the levels at 7 days. This demonstrates that both treatments have an inhibitory effect on the plasma ET-1 level, which inhibits pulmonary fibrosis development. The Schisandrae group and the combination therapy group showed no significant difference from the model group in plasma ET-1 levels $(\mathrm{P}>0.05)$, which indicates that these two drugs are less effective than Ephedra and hydrocortisone.

The theory of ascending-descending-floating-sinking is an important pillar to support the use of traditional Chinese medicine and has been continually developed and improved. This theory may be used as a guide for the treatment of lung diseases. Its value requires the confirmation of three points: the physiological characteristics of the lungs, the characteristics of pathogenesis and disturbance in ascending and descending airflow in lung disease, and the drug properties of traditional Chinese medicine. Therefore, this theory is important for the understanding and treatment of lung disease, including pulmonary fibrosis, and merits serious consideration in IPF research.

\section{ACKNOWLEDGMENTS}

Research supported by the Natural Science Foundation of China (\#81373887), the China Postdoctoral Science Foundation (\#2012M510346), and the Beijing University of Chinese Medicine Innovation Team project funding.

\section{References}

Hirose S, Hosoda Y, Furuya S, Otsuki T, et al. (2000). Expression of vascular endothelial growth factor and its receptors correlates closely with formation of the plexiform lesion in human pulmonary hypertension. Pathol. Int. 50: 472-479.

Keane MP, Donnelly SC, Belperio JA, Goodman RB, et al. (2002). Imbalance in the expression of CXC chemokines correlates with bronchoalveolar lavage fluid angiogenic activity and procollagen levels in acute respiratory distress syndrome. J. Immunol. 169: 6515-6521.

Li H and Li GQ (2009). Clinical observation of Yi Qi Huo Xue Tong Luo Tang treated pulmonary fibrosis. Chin. J. Inform. Tradit. Chin. Med. 16: 65-66.

Lohmann-Matthes ML, Steinmuller C and Franke-Ullmann G (1994). Pulmonary macrophages. Eur. Respir. J. 7: 16781689.

Lu S, Zhang N and Zhang X (2001). mRNA expression of matrix metalloproteinase-2 and membrane type matrix metalloproteinase in pulmonary fibroblasts and macrophages of rat pulmonary fibrosis model. Zhonghua Jie He He Hu Xi Za Zhi. 24: 527-530.

Meltzer EB and Noble PW (2008). Idiopathic pulmonary fibrosis. Orphanet. J. Rare. Dis. 3: 8.

Rubins JB (2003). Alveolar macrophages: wielding the double-edged sword of inflammation. Am. J. Respir. Crit. Care Med. 167: 103-104. 
Snider GL, Celli BR, Goldstein RH, O’Brien JJ, et al. (1978). Chronic interstitial pulmonary fibrosis produced in hamsters by endotracheal bleomycin. Lung volumes, volume-pressure relations, carbon monoxide uptake, and arterial blood gas studied. Am. Rev. Respir. Dis. 117: 289-297.

Song C and Liu SX (2007). Lian Fei Zhi Ke Yin treated 43 cases of pulmonary fibrosis. Modern Tradit. Chin. Med. $27: 14$.

Strieter RM, Belperio JA and Keane MP (2002). CXC chemokines in angiogenesis related to pulmonary fibrosis. Chest 122: 298S-301S.

Syeda F, Grosjean J, Houliston RA, Keogh RJ, et al. (2006). Cyclooxygenase-2 induction and prostacyclin release by protease-activated receptors in endothelial cells require cooperation between mitogen-activated protein kinase and NF-kappaB pathways. J. Biol. Chem. 281: 11792-11804.

Szapiel SV, Elson NA, Fulmer JD, Hunninghake GW, et al. (1979). Bleomycin-induced interstitial pulmonary disease in the nude, athymic mouse. Am. Rev. Respir. Dis. 120: 893-899.

Tzortzaki EG, Antoniou KM, Zervou MI, Lambiri I, et al. (2007). Effects of antifibrotic agents on TGF-beta1, CTGF and IFN-gamma expression in patients with idiopathic pulmonary fibrosis. Respir. Med. 101: 1821-1829.

Unemori EN, Pickford LB, Salles AL, Piercy CE, et al. (1996). Relaxin induces an extracellular matrix-degrading phenotype in human lung fibroblasts in vitro and inhibits lung fibrosis in a murine model in vivo. J. Clin. Invest. 98: $2739-2745$

Weidner N (1995). Intratumor microvessel density as a prognostic factor in cancer. Am. J. Pathol. 147: 9-19.

Yao CF and Jiang SL (2005). Clinical observation of Liu An Qi Wei Jian treated 32 cases of pulmonary fibrosis. J. Sichuan Tradit. Chin. Med. 23: 52-53.

Ye Q, Dalavanga Y, Poulakis N, Sixt SU, et al. (2008). Decreased expression of haem oxygenase-1 by alveolar macrophages in idiopathic pulmonary fibrosis. Eur. Respir. J. 31: 1030-1036.

Zhai HQ, Yuan Y, Ou M and Zheng HZ (2010). Influence of Yifei Huoxue particles on pulmonary vascular injury pathology in the chronic pulmonary heart disease in rabbits. Zhongguo Bing Li Sheng Li Za Zhi 26: 590-592.

Zhao YS and Yin SP (2008). Yi Qi Huo Xue Tang treated 21 cases of pulmonary fibrosis. J. Shanxi Tradit. Chin. Med. 29: 391-392.

Zheng R, Xu LY and Kang J (2000). Pulmonary fibrosis in rats? 1 mRNA expression of transforming growth factorinduced changes in the timing and number. Zhonghua Nei ke Za Zhi 39: 621-622. 